\title{
Recent patent applications in antivirals
}

Patent \#

EP 1674104 WO 2006067606 EP 1827457

JP 2006306836 WO 2007080669

A composition comprising a phenol derivative or its salt as an active ingredient; useful for treating bacterial and viral infections in pharmaceuticals, agrochemicals and as a disinfectant.

US 20070099296 A method of determining whether a test hepatitis C virus (HCV) has an altered susceptibility to a compound, comprising contacting a test host cell with the compound and detecting the activity of the indicator gene.

US 20070087434, An antiviral artificial cell comprising an artificial cytoskeleton JP 2007091671 comprising an electromagnetic wave absorber that generates heat to neutralize a virus when externally irradiated with an electromagnetic wave, an artificial cytomembrane wrapping the artificial cytoskeleton and a nanoparticle having a magnetic spin, rotatably retained on the artificial cytomembrane and having a surface for capturing a virus. Useful as an antiviral drug used in a biofilter for removing virus.

US 20060142298, New aluminium salts of 1,2,3-triazoles useful for treating infection WO 2006071564, by human immunodeficiency virus.

US 7183284

JP 2006335756

A medical treatment method, or antiviral drug or riboflavin (vitamin B2) drug for viral infection, preferably AIDS caused by HIV, having an envelope, involving using diethyl ether or a substance such as pancreas lipase, bile acid, chitosan, acetone, ethanol, newlase etc. where the concentration of the substance is not altered. contact lenses.

\begin{tabular}{|c|c|c|c|}
\hline Assignee(s) & Inventor(s) & $\begin{array}{c}\text { Priority } \\
\text { application date }\end{array}$ & $\begin{array}{c}\text { Publication } \\
\text { date }\end{array}$ \\
\hline $\begin{array}{l}\text { CNRS (Paris), } \\
\text { INSERM (Paris) }\end{array}$ & $\begin{array}{l}\text { Agrofoglio L, } \\
\text { Amblard F, } \\
\text { Aucagne V, } \\
\text { Durantel D, } \\
\text { Escuret V, } \\
\text { Joubert N, } \\
\text { Trepo C, } \\
\text { Zoulim F }\end{array}$ & $12 / 24 / 2004$ & $\begin{array}{l}\text { 6/28/2006, } \\
\text { 6/29/2006, } \\
\text { 9/5/2007 }\end{array}$ \\
\hline $\begin{array}{l}\text { Micro Biotech } \\
\text { (Tokushima, } \\
\text { Japan) }\end{array}$ & $\begin{array}{l}\text { Higuchi M, } \\
\text { Higuchi N, } \\
\text { Higuchi T, } \\
\text { Koyama H, } \\
\text { Shibata H }\end{array}$ & $1 / 11 / 2005$ & $\begin{array}{l}\text { 11/9/2006, } \\
7 / 19 / 2007\end{array}$ \\
\hline $\begin{array}{l}\text { Monogram } \\
\text { Biosciences } \\
\text { (South San } \\
\text { Francisco, CA, } \\
\text { USA) }\end{array}$ & $\begin{array}{l}\text { Gamarnik A, } \\
\text { Parkin NT }\end{array}$ & 7/30/1997 & $5 / 3 / 2007$ \\
\hline Toshiba (Tokyo) & Itaya $\mathrm{K}$, Naruse $\mathrm{Y}$ & 9/29/2005 & $\begin{array}{l}4 / 19 / 2007 \\
4 / 12 / 2007\end{array}$ \\
\hline
\end{tabular}

\begin{tabular}{llcc} 
Bristol-Myers Kadow JF, & $12 / 29 / 2004$ & $6 / 29 / 2006$, \\
Squibb (Princeton, Regueiro-Ren A & & $7 / 6 / 2006$, \\
NJ, USA), & & $2 / 27 / 2007$ \\
$\begin{array}{l}\text { Kadow JF, } \\
\text { Regueiro-Ren A }\end{array}$ & & \\
\hline $\begin{array}{l}\text { Uchiyama M Uchiyama M } \\
\text { 6/6/2005 }\end{array}$ & $12 / 14 / 2006$
\end{tabular}

Uchiyama M

$6 / 6 / 2005$

$2 / 14 / 2006$
WO 2006091798 A method for lowering viral load of a virus, where the virus causes a chronic viral infection and is resistant to an antiviral drug, comprising administering to a host a medicament comprising the antiviral drug, where the antiviral drug is capable of selecting for a predetermined antiviral drug-resistant mutation in a viral protein, thus creating an antiviral drug-resistant virus; and a synthetic peptide of 9-15 amino acids, comprising the antiviral drug resistant mutation in the viral protein, where the peptide induces an antiviral cytotoxic T lymphocyte response.

WO 2006084275, A method for making a system for delivering drugs (e.g., antibiotic, US 20060177483 anti-inflammatory, antihistamine, antiviral agent, cancer drug or anesthetic) involving forming a recognitive polymer hydrogel into
US Department

of Health and

Human Services

(Washington, DC

USA
Berzofsky

JA, Broder S

Catanzaro A,

Snyder JT,

Yarchoan R
2/22/2005 8/31/2006

$\begin{array}{ll}\text { (Auburn, AL, } & \text { Venkatesh S } \\ \text { USA), Byrne ME, } & \text { Ewing WR, }\end{array}$

$2 / 4 / 2005$

$8 / 10 / 2006$

Venkatesh S, Huang Y,

Ewing WR, Mikkilineni A

Huang $Y, \quad$ Sitkoff $D F$,

Mikkilineni A, Sun C, Yu G

Sitkoff DF,

Sun C, Yu G

WO 2006077427 A combination useful for treating infections caused by a glycosylated envelope protein bearing virus, comprising a viral entry inhibitor and an adjunctive agent selected from glycosylation modulator, alkovir and glycovir (e.g., glucovir).

WO 2006065125 A method for determining whether a compound (e.g., an antiviral drug) can be transported through active transport by breast cancer resistance protein (BCRP) into the milk or colostrum of a lactating mammal, by determining whether the compound is a BCRP substrate.
Carroll MW,

(Ceredigion, UK) Nash RJ,

Slingsby JH

Schinkel AH,

van Herwaarden $\mathrm{AE}$
Het Nederlands Jonker JW,

Source: Thomson Scientific Search Service. The status of each application is slightly different from country to country. For further details, contact Thomson Scientific, 1800 Diagonal Road, Suite 250, Alexandria, VA 22314, USA. Tel: 1 (800) 337-9368 (http://www.thomson.com/scientific). 